\title{
Superconductor to normal-metal transition in finite-length nanowires: Phenomenological model
}

\section{Citation}

Refael, Gil, Eugene Demler, and Yuval Oreg. 2009. “Superconductor to Normal-Metal Transition in Finite-Length Nanowires: Phenomenological Model." Physical Review B 79 (9). https:// doi.org/10.1103/physrevb.79.094524.

\section{Permanent link}

http://nrs.harvard.edu/urn-3:HUL.InstRepos:41412123

\section{Terms of Use}

This article was downloaded from Harvard University's DASH repository, and is made available under the terms and conditions applicable to Other Posted Material, as set forth at http:// nrs.harvard.edu/urn-3:HUL.InstRepos:dash.current.terms-of-use\#LAA

\section{Share Your Story}

The Harvard community has made this article openly available.

Please share how this access benefits you. Submit a story.

Accessibility 


\title{
Superconductor to normal-metal transition in finite-length nanowires: Phenomenological model
}

\author{
Gil Refael, ${ }^{1}$ Eugene Demler, ${ }^{2}$ and Yuval Oreg ${ }^{3}$ \\ ${ }^{1}$ Department of Physics, California Institute of Technology, MC 114-36, Pasadena, California 91125, USA \\ ${ }^{2}$ Department of Physics, Harvard University, 17 Oxford Street, Cambridge, Massachusetts 02138, USA \\ ${ }^{3}$ Department of Condensed Matter, Weizmann Institute of Science, Rehovot 76100, Israel
}

(Received 21 November 2008; published 27 March 2009)

\begin{abstract}
In this paper we discuss the interplay of quantum fluctuations and dissipation in uniform superconducting nanowires. We consider a phenomenological model with superconducting and normal components and a finite equilibration rate between these two fluids. We find that phase-slip dipoles proliferate in the wire and decouple the two fluids within its bulk. This implies that the normal fluid only couples to the superconductor fluid through the leads at the edges of the wire, and the local dissipation is unimportant. Therefore, while long wires have a superconductor-metal transition tuned by local properties of the superconducting fluid, short wires have a transition when the total resistance is $R_{\mathrm{tot}}=R_{Q}=h / 4 e^{2}$.
\end{abstract}

DOI: 10.1103/PhysRevB.79.094524

PACS number(s): 74.81.Fa, 74.78.Na

\section{INTRODUCTION}

Quantum phase transitions have long been at the forefront of condensed-matter theory. Especially interesting are systems of reduced dimensionality and size, where fluctuations are enhanced, and ordering is illusive, and far from being explained by mean field theory. Such systems exhibit a surprising degree of universality; for instance, as observed in Refs. 1 and 2, a mesoscopic Josephson junction shunted by a resistor $R$ undergoes a (so-called Schmid) transition between Coulomb-blockade (normal) and superconducting phases when the shunt resistor is $R=R_{Q}=h / 4 e^{2}=6.45 \mathrm{k} \Omega .^{3-5}$ Fluctuations of the superconducting phase angle, i.e., phase slips, induce this transition; they also control the onset of superconductivity in long thin wires and Josephson junction chains, ${ }^{6-10}$ where the competition between local charging energy, which creates phase slips, and the superconducting stiffness tunes the transition. ${ }^{11-13}$

Our focus is on the experiments on $\mathrm{Mo}_{79} \mathrm{Ge}_{21}$ (amorphous) nanowires as narrow as $5-15 \mathrm{~nm}$. Resistance vs temperature curves showed a transition between superconducting (resistance decreasing upon cooling) and normal, or weakly insulating, (resistance nondecreasing upon cooling) behaviors. A first set of measurements on wires of various diameters and lengths $100 \mathrm{~nm}<L<200 \mathrm{~nm}$, showed a remarkable result: a transition when the total resistance of the wire was $R_{Q}=h / 4 e^{2}=6.45 \mathrm{k} \Omega,{ }^{14}$ as if the entire wire was a single shunted junction. But the coherence length of MoGe is $\xi \sim 20 \mathrm{~nm}$; thus the wire should differ dramatically from a single junction. Indeed, later experiments on longer wires, $200 \mathrm{~nm}<L<1000 \mathrm{~nm}$, showed a weak transition that depended on the resistance per length or cross section of the wires, i.e., on a local quantity, rather than the total resistance. ${ }^{15} \mathrm{~A}$ third set of experiments ${ }^{16}$ could neither prove nor disprove the global nature of the transition in the shorter wires. The nature of the breakdown of superconductivity in ultrathin MoGe nanowires thus remains an open question. Below we address this question and also consider the general interplay between supercondcutivity and dissipation in such nanoscale superconductors.

\section{A. Overview of results}

In this paper, we describe nanowires using a two-fluid model, which assumes that Cooper pairs couple to a normal electron fluid, which provides local dissipation (Fig. 1). Remarkably, we find that at sufficiently low temperature, the normal and superconducting fluids within a continuous nanowire decouple due to quantum phase fluctuations, thus rendering the local dissipation unimportant. Therefore, the superconducting degrees of freedom can only couple to the dissipative normal fluid at the leads, on the edges of the wire, where they couple to its total normal-state resistance, $R_{\mathrm{tot}}$. As a result, we show that indeed short wires may undergo a global dissipative Schmid transition tuned by the total wire resistance when $R_{\mathrm{tot}}=R_{Q}$. In short, we mean wires with length $L>\xi$, but shorter than the thermal length, $L<\hbar u / T$ (with $u$ as the Mooij-Schön phase velocity and $T$ being the lowest temperature in the experiment). ${ }^{17}$ The global dissipative Schmid transition also requires that the resistance of the wire is, at most, in the range of the quantum resistance, while the thickness of the wire is not so large that phase slips are completely suppressed. This requires that $R_{\xi} / R_{Q}>c$, with $c$ $\sim 0.1$, which translates to the requirement $L<\xi / c .{ }^{18}$ After our work was completed, this result was verified in MoGe wires with $50 \mathrm{~nm}<L<300 \mathrm{~nm}^{19}$

Our results follow from the analysis in Ref. 20 where infinite length nanowires were analyzed. Below we sketch the development of the two-fluid model, show how phaseslip dipoles decouple the normal and superconducting fluids,

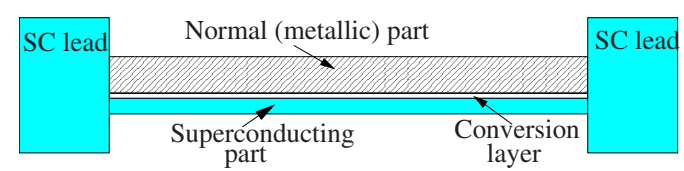

FIG. 1. (Color online) A two-fluid model of a superconducting nanowire with a normal part, depicted as a separate region. In order for electrons to change from normal to superconducting, they need to pass through the conversion layer, which has a finite conductivity. Proliferated phase-slip dipoles inhibit two-fluid relaxation and make the conversion layer insulating, which renders the normal part as an effective single shunt resistor. 
a.

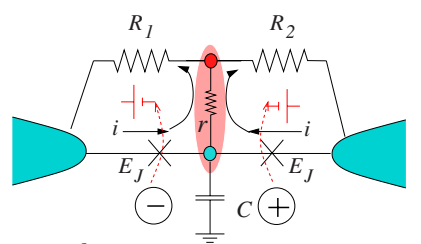

b

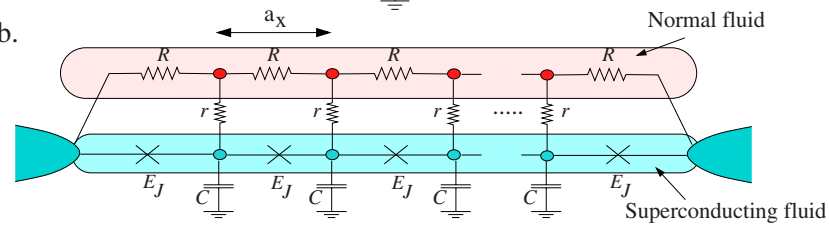

FIG. 2. (Color online) (a) Single two-fluid grain (pink ellipse) between two leads: dipoles produce two opposing voltage spikes on the two junctions, which oppose super-to-normal conversion currents leaving the superconducting part of the grain (bottom circle in ellipse) and entering the normal part (top circle). When dipoles proliferate, the superfluid-normal conversion resistance (and time) effectively diverges, $r \rightarrow \infty$, and the normal and superfluid are completely decoupled. (b) We begin our study with a chain of two fluid grains, which is a discretized version of the nanowire in Fig. 1. Note that $1 / r=\Upsilon a_{x}$ and $R=a_{x} \rho$.

and apply the model to finite nanowires to allow a direct comparison with the experiment.

\section{TWO-FLUID MODEL AND DYNAMIC DECOUPLING BETWEEN THE NORMAL-FLUID AND SUPERFLUID}

\section{A. Two fluid model}

The hint of a Schmid transition, the long resistive tails seen in experiments, and the strong disorder of the MoGe nanowires suggest the presence of local dissipation, which motivates a two-fluid model approach to the nanowire problem. We assume that charge can flow in the nanowires in two ways: as diffusive normal electrons with resistivity $\rho$-normal fluid-and as bosonic Cooper pairs-superfluid. The normal fluid stems from strong disorder and phase fluctuations, which suppress the proximity effect and possibly give rise to normal regions and a finite density of states for single electrons at the Fermi level. The two fluids can have a different chemical potential and can exchange charge with a finite, bare, relaxation time, $\tau_{r}=\Upsilon^{-1}$, in a bulk system [see Figs. 1 and 2(b) for a discrete model]. This is related to the branch imbalance relaxation time, ${ }^{21,22}$ first measured by Clarke $^{23}$ in Sn wires.

Before plunging to the analysis, note that earlier works on similar models considered only the perfect normal fluidsuperfluid coupling, $Y=\infty$ case, and found a superconducting-metal transition tuned by the resistance per length, ${ }^{11-13,24}$ as did Refs. 25 and 26. Alternative approaches assumed external dissipation coupled to the leads but not to the bulk of the wire ${ }^{27}$, or discussed the onset of superconducting correlations and neglected phase fluctuations. ${ }^{28}$

Indeed, in our model as well, sufficiently long but finite wires should exhibit a superconductor (SC)-normal crossover tuned by their cross-section area, which sets the bare fugacity of quantum phase slips, ${ }^{18}$ as well as their stiffness. But quantum fluctuations in the form of phase-slip dipoles make the Cooper pair to normal-electron conversion rate vanish at $T=0$ in the bulk of the nanowire: $Y \rightarrow 0$. As claimed above, this leads to a true Schmid transition for short wires, which effectively become a short dissipationless superconducting wire, shunted through the leads by the total normal-state resistance $R_{T}$.

\section{B. Dynamic decoupling in a two-junction system}

The crucial two-fluid decoupling is already evident in a simple two-junction system [Fig. 2(a)]..$^{29,30}$ The grain contains superfluid electrons, which couple to the leads via Josephson junctions, and normal electrons, which couple to the leads through the shunt resistors. When $r=0$ (i.e., vanishing conversion resistance between the two fluids), the two junctions in the system are independent of the dc limit. Phase slips-events where the phase across a Josephson junction tunnels by $2 \pi$-create a sudden voltage drop that opposes any supercurrent flowing and thus induce dissipation. A Schmid transition occurs in each junction when $R_{i}=R_{Q}(i$ $=1,2)$. When $r>0$, the two junctions become coupled, and phase slips may form bound dipoles: simultaneous phase slip and antiphase slip in the two junctions. Remarkably, dipoles do not destroy the coherence between the two leads since they produce equal and opposite voltage drops. Nevertheless, as single phase slips block supercurrents across their Josephson junctions when they proliferate, dipoles block the normal-superfluid conversion channel in the grain: a conversion current $2 i$ [Fig. 2(a)] flowing across $r$, with no lead-tolead current, implies a current $i$ on both junctions but in opposite directions. $i$ couples directly to the voltage drop of the dipoles; when proliferated, they block this current mode and thus decouple the normal fluid and superfluid. Phase slip dipoles proliferate roughly when $r>R_{Q}$. In this case a global Schmid transition takes place when $R_{1}+R_{2}=R_{Q}$.

\section{Dynamic decoupling of the two fluids in a Josephson junction array}

Next, we summarize how the normal super fluids decouple in infinite wires, first using a discrete model [Fig. $1(b)]$ and then taking its continuum limit. ${ }^{20}$ Starting with an infinite chain of mesoscopic two-fluid grains [Fig. 1(b)], ${ }^{20}$ the low-energy action for the chain is given in terms of a $2 d$ gas of phase slips with the following two-body interactions: with two phase slips separated in space by $x$ and in imaginary time by $\tau$, their interaction is

$$
p_{1} p_{2}\left(K \log \frac{a_{\tau}}{\sqrt{x^{2} / u^{2}+\tau^{2}}}+\alpha e^{-|x| / \lambda} Q \log \frac{a_{\tau}}{|\tau|}\right) .
$$

$u=a_{x} \sqrt{E_{J} E_{C}} / \hbar$ is the Mooij-Schön velocity, ${ }^{31}$ and $a_{\tau}=a_{x} / u$. $p_{i}= \pm$ is the phase slip polarity. The first term is the usual isotropic interaction of a $1+1 X Y$ model due to the plasmons in the Josephson junction array; $K=2 \pi \sqrt{E_{J} / E_{C}}$ and $E_{C}$ $=(2 e)^{2} / C$. The second term is due to the dissipative interaction: $\alpha=\max \left\{\frac{R_{Q}}{\sqrt{r R}}, \frac{R_{Q}}{R}\right\}$ and $\lambda_{Q}=\max \left\{a_{x} \sqrt{r / R}=1 / \sqrt{\Upsilon \rho}, a_{x}\right\}$ is a new length scale that arises from the two-fluid finite relaxation time. As in the two-junction case, dipoles must be explicitly included in the low-energy description of this 
model. ${ }^{12,13,20}$ We denote the fugacity of single phase slips as $\zeta$, and the fugacity of a dipole with moment $n$ as $\eta_{n}$. For completeness, we quote here the explicit field theory for the infinite chain,

$$
\begin{gathered}
\int \frac{d \omega d k}{(2 \pi)^{2}}\left[\left(u k^{2}+\frac{1}{u} \omega^{2}\right) \frac{\theta_{(k, \omega)}^{2}}{4 \pi K}+\frac{r}{4 \pi R_{Q}}|\omega|\left(k^{2}+\frac{R}{r}\right) \psi_{(k, \omega)}^{2}\right] \\
-\int d \tau \sum_{i}\left[\zeta \cos \left(\theta_{i}+\psi_{i}\right)+\eta_{n} \cos \left(\Delta_{n} \theta_{i}+\Delta_{n} \psi_{i}\right)\right]
\end{gathered}
$$

with $u=a_{x} \sqrt{E_{J} E_{C}} / \hbar$ and $\theta$ and $\psi$ mediating the plasmon and dissipative interactions, respectively. At high energies $\eta_{n}$ $\sim \zeta^{2}$ and $\Delta_{n} f_{i}=f_{i+n}-f_{i}$. This is a representation dual to the SC phase representation; a path integral in terms of the fields $\psi$ and $\theta$ will give the partition function of phase slips with the interaction in Eq. (1) in the grand canonical ensemble as a power series in $\zeta$ and the $\eta_{n}$ 's. Hence $\exp \left(i \psi_{i}+i \theta_{i}\right)$ is interpreted as the operator that creates a phase slip on junction $i$.

It is useful to compare the relatively complicated interaction between phase slips in an infinite chain with that of phase slips in a single Josephson junction. In a single junction the interaction is $p_{1} p_{2} \frac{R_{Q}}{R} \log \frac{a_{\tau}}{|\tau|}$. The Schmid transition, which marks phase-slip proliferation, occurs when the gain in entropy due to separating a phase-slip from an antiphase slip, $S=\log \left(a_{\tau} T\right)$, equals the required interaction energy, $\frac{R_{Q}}{R} \log \left(a_{\tau} T\right)$. Employing the same argument for the two-fluid Josephson chain yields the approximate SC-normal phase boundary,

$$
K+\frac{1}{2} \alpha \sim 4 .
$$

This transition is essentially the $1+1$ Kosterlitz-ThoulessBerezinski (KTB) transition of the Josephson junction array in accordance with Ref. 11 (for a more refined analysis see Ref. 20). But this argument, as well as the interactions in Eq. (1), ignores phase-slip dipoles. When dipoles are proliferated, the normal fluid and superfluid decouple, and the superconducting part of the wire exhibits a SC-normal KTB transition when $K \sim 4$. Phase-slip dipoles, we find, proliferate when

$$
\frac{2 R_{Q}}{R}\left(1-e^{-a_{x} / \lambda} Q\right)<1
$$

The left-hand side is the strength of dipole interaction, which consists of the self-interaction of the slip and antislip, and also their mutual interaction. $a_{x}$ is the distance between grains in the model, in accordance with Eq. (1).

\section{Two fluid model and phase slips in the continuum limit}

In the continuum limit, dipoles always proliferate and cut off the superfluid-normal conversion. The continuum limit implies that the distance between grains is $a_{x} \rightarrow 0$; but this makes a Josephson junction (and therefore also a phase slip on a junction) shrink to length zero as well. A phase slip occurring on physical nanowires, however, has a character-

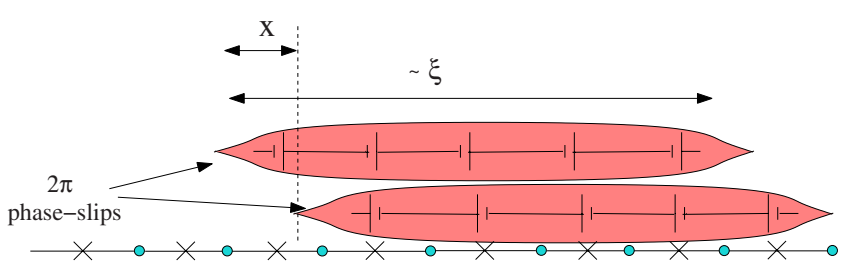

FIG. 3. (Color online) To describe a continuous wire, we take the limit $a_{x} \rightarrow 0$ while keeping the coherence length and size of phase slip, $\xi$, fixed. This implies that phase slips are spread over $\xi / a_{x}$ junctions and can form dipoles with separation $x<\xi$. The voltage signs symbolize the voltage drop caused by a phase slip.

istic length $\xi$ (coherence length). This can be simply reconciled by allowing phase slips to smoothly spread over $\sim \xi / a_{x}$ junctions. $^{20}$

Technically, we transform the zeta term in Eq. (2) as $\cos (\psi+\phi)_{(x, \tau)} \rightarrow \cos \frac{\xi}{a_{x}} \sum_{r} f(r)(\psi+\phi)_{(x+r, \tau)}$, where $f(r)$ is a smooth, normalized, function centered around 0 , with width $\xi . f(r)$ can also be understood as the "charge" distribution of a phase slip in space and can be used directly in Eq. (1). Two phase slips centered around 0 and $x$, separated by imaginary time $\tau$, will thus interact as

$$
\begin{aligned}
& p_{1} p_{2} \int d x_{1} \int d x_{2} f\left(x_{1}-0\right) f\left(x_{2}-x\right) \\
& \quad \times\left(K \log \frac{a_{\tau}}{\sqrt{\left(x_{1}-x_{2}\right)^{2} / u^{2}+\tau^{2}}}+\alpha e^{-\left|x_{1}-x_{2}\right| / \lambda} Q \log \frac{a_{\tau}}{|\tau|}\right) .
\end{aligned}
$$

We similarly treat the dipole $\eta_{n}$ terms. The smearing reflects that in nanowires, phase slips can have an almost arbitrary overlap, $\Delta<\xi$ (Fig. 3), with other phase slips. This procedure is valid since each phase slip produces dissipation in the normal electron channel. The shortest length scale that can be resolved by the normal electrons is their Fermi wavelength, which is $\lambda_{F} \ll \xi$. $\lambda_{F}$ is therefore the correct UV cutoff for the dissipative portion of the interaction in Eqs. (1) and (5). Thus, so long that $a_{x}>\lambda_{F}$, we must consider a phase slip as an extended object.

Let us now apply the understanding of the continuum limit to dipoles and the condition for their proliferation. The continuum generalization of Eq. (4) is that dipoles proliferate when $\frac{R_{Q}}{R_{\xi}}\left(\frac{\Delta}{\max \left\{\lambda_{O}, \xi\right\}}\right)^{2}<1$ (where $R_{\xi}=R_{\text {tot }} \xi / L=\rho \xi$ ). Thus, for any $R_{\xi}, r$ there is a separation $\Delta_{c}$ below which dipoles proliferate. By incorporating the above analysis and the appropriate screening terms in Eq. (2) we see that the normalsuperfluid conversion is cut off at temperatures (or frequencies) $T \sim \zeta_{0}^{2} a_{\tau}$, where $\zeta_{0}$ is the bare fugacity of phase slips and $a_{\tau}^{-1} \sim(\xi / c)^{-1}$ is the UV cutoff in Eq. (2).

\section{SUPERCONDUCTING-NORMAL TRANSITION IN A FINITE NANOWIRE}

Above we showed that phase slip dipoles always proliferate at a continuous nanowire. A prime purpose in this paper is the application of the above insights to a finite continuous wire. Phase slips exhibit an interaction due to the plasma 
a.

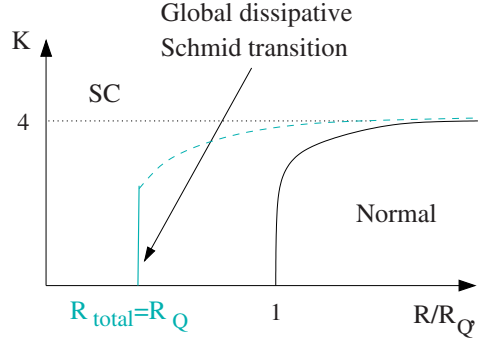

b. $\mathrm{L} / \mathrm{R}$ total $[\mathrm{nm} / \mathrm{k} \Omega]$

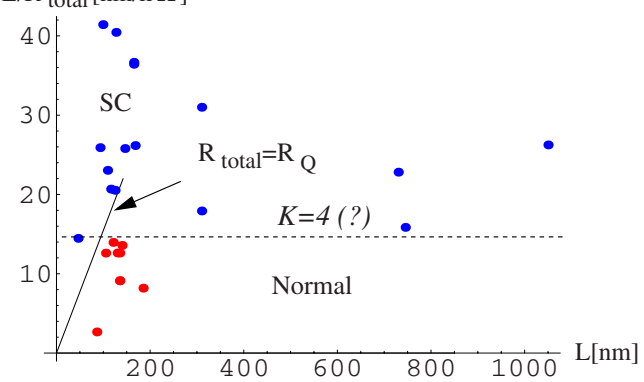

FIG. 4. (Color online) (a) Phase diagram of the two-fluid chain and wire. The black line describes (roughly) the SC-normal boundary of an infinite two-fluid chain [Fig. 2(b)], with $r<R_{Q}$; both $K$ $=2 \pi \sqrt{E_{J} / E_{C}}$ and $R$ are local quantities. When $r>R_{Q}$, normalsuperfluid conversion is cut off, the local dissipation becomes unimportant, and only the horizontal line applies. In finite continuous wires (gray line), the transition takes a dissipative nature when the wire is short and occurs when $R_{\text {tot }}=R_{Q}$; longer wires have a local crossover-dashed line-tuned by the superconducting stiffness $\sim K$. (b) Phase diagram for MoGe nanowires in Refs. 14-16. The $y$ axis, $L / R_{\mathrm{tot}} \propto A / \rho_{\mathrm{MoGe}}$, is proportional to the surface area, which is proportional to $K$. The $x$ axis is the length. The blue dots are insulating samples, whereas the red dots are superconducting. The horizontal dashed line marks the long-wire crossover, while the diagonal black line marks the transition line $R_{\mathrm{tot}}=R_{Q}$.

waves in the chain, $K \log \frac{a_{\tau}}{\sqrt{x^{2} / u^{2}+\tau^{2}}}$ and also due to the dissipation through the normal resistance. But now, looking back at Fig. 2(b), if the conversion channel in the wire is blocked, i.e., $r \rightarrow 0$, the only way in which the superfluid couples to the dissipation is through the leads. Therefore, the conclusion from the above considerations is that a finite nanowire is effectively described by a chain of Josephson junctions, shunted by the global resistance in the chain since proliferated dipoles decouple the normal and superfluid electrons. The resulting dissipative interaction between phase slips is

$$
\frac{R_{Q}}{R_{\mathrm{tot}}} \log \frac{a_{\tau}}{|\tau|}
$$

The same consideration that led to Eq. (3) now indicates that a transition will now occur when

$$
K+\frac{R_{Q}}{R_{\text {tot }}} \sim 4
$$

[see Fig. 4(a)]. At closer consideration, however, the finite size of the wire makes the first term in Eq. (1), which is the $K$ dependent logarithmic interaction be cut off at times $\tau$ $>L /(\hbar u)$. Therefore, the finite size of the wire turns any
KTB transition tuned by $K$ into a crossover, and no quantumcritical scaling is observed for lengths $L<u / \hbar T$. $K$ may still have a strong effect on the behavior of the wire also due to the bare fugacity of phase slips being exponentially suppressed with $K$, which will also seem like a crossover tuned by $K$. The second term in Eq. (1), however, becomes the logarithmic interaction in Eq. (6), and hence the total resistance tunes a Schmid transition when $R_{\mathrm{tot}}=R_{Q}$ [see Fig. 4(a)].

\section{COMPARISON WITH EXPERIMENTS}

Our conclusions could be easily related to the nanowire experiments. ${ }^{14-16,32}$ In long wires, we expect a SC-normal crossover tuned by stiffness (as in Refs. 11 and 24), but in short wires, we expect a Schmid transition tuned by the total normal-part resistance. In Fig. 4(b) we recast the diagram of Fig. 4(a) for the MoGe nanowire experiments, plotting $L / R_{\mathrm{tot}} \propto A$ vs $L$, with $L$ as the length of the wire and $A$ its cross section area. The diagonal line marks $R_{\mathrm{tot}}=R_{Q}$. Above it we expect $T=0$ superconductivity. The horizontal line marks the SC-normal crossover in longer wires. This line most probably arises from the exponential dependence of the bare quantum-phase-slip fugacity on thickness ${ }^{18}$ but may also be associated with a KTB transition at $K \sim 4$ or a fermionic $T_{C}$ suppression mechanism, which also depends on $R_{\xi} / R_{Q} \cdot{ }^{26}$ After completing the analysis described here, Bezryadin and co-workers ${ }^{16,32,33}$ measured a large number of short samples with $L<150 \mathrm{~nm}$. These show near perfect fit with our prediction of a universal transition at $R_{\mathrm{tot}}=R_{Q}$ for shorter wires.

The application of our simple theory to the nanowire experiments requires several caveats. (1) It is natural to associate the resistance of the nanowire devices at temperatures just below the SC transition of the leads, with the total normal-part nanowire resistance, $R_{\text {tot }}$. It is unclear, however, how this resistance is related to the normal-state resistance of the nanowires at temperatures above the bulk critical temperatures for MoGe. (2) In addition, the origin and precise nature of normal electrons in the wires are unknown. Possibly, phase fluctuations or the strong disorder stifle the proximity effect and give rise to a normal part. Particularly, if the normal-superconductor relaxation rate is indeed suppressed, each phase slip gives rise to a long-lived population of quasiparticles, as is the case in Ref. 34 where dissipation at phase-slip centers is investigated. (3) The resistance vs temperature curves measured by Bezryadin on the superconducting side show sharp exponential, activated-like, decay of the resistance, contrary to a naive quantum phase-slip theory, where an algebraic dependence of the resistance on temperature is expected. Similarly, the wires remaining normal show a weakly insulating behavior, with a charge gap that corresponds to the Coulomb blockade of the leads. ${ }^{32}$ These observations do not contradict the possibility of a Schmid transition and can be understood by also considering the effective dissipation produced by a finite density of phase slips, which is too large to justify the perturbative analysis pursued here. Such considerations appeared to describe the intermediatecoupling regime of the two-junction system. ${ }^{30}$ Also, using the results presented here, which establish the existence of 
the Schmid transition, we show in Refs. 35 and 36 that a nonsparse density of phase slips in a short chain of Josephson junctions indeed gives rise to a sharp temperature dependence, which agrees well with experiments.

\section{CONCLUSIONS}

The main result of this paper is the divergence of the normal-superfluid relaxation time, $\tau_{r}=\Upsilon^{-1}$, in continuous uniform nanowires due to quantum fluctuations. Apart from the direct application of our theory to the MoGe nanowire experiments, this effect could be directly investigated in mesoscopic and nanoscopic systems where quantum fluctuations are apparent at relatively high temperatures. Some early experiments in this direction on nanostructures not uniform enough, but with quantum fluctuations, are described in Ref. 37. In future work we hope to address the issues of the origin and nature of the normal part in nanowires, and its interplay with phase-slip density, and the diverging relaxation time.

\section{ACKNOWLEDGMENTS}

The authors thank D. S. Fisher, who collaborated with them on much of the reported research. They also thank A. Bezryadin, A. Bollinger, P. Goldbart, B. I. Halperin, D. Meidan, D. Podolsky, and D. Shahar for many helpful discussions. G.R. thanks the Technion ITP and the Weizmann Institute for their generous hospitality. Y.O. acknowledges support of the ISF.
${ }^{1}$ J. S. Penttilä, Ü. Parts, P. J. Hakonen, M. A. Paalanen, and E. B. Sonin, Phys. Rev. Lett. 82, 1004 (1999).

${ }^{2}$ M. Watanabe and D. B. Haviland, in Studies of High Temperature Superconductors (Advances in Research and Applications), edited by A. Narlikar (Nova Science, New York, 2002), Vol. 43, Chap. 6.

${ }^{3}$ A. Schmid, Phys. Rev. Lett. 51, 1506 (1983).

${ }^{4}$ S. Chakravarty, Phys. Rev. Lett. 49, 681 (1982).

${ }^{5}$ S. A. Bulgadaev, JETP Lett. 39, 315 (1984).

${ }^{6}$ P. Xiong, A. V. Herzog, and R. C. Dynes, Phys. Rev. Lett. 78, 927 (1997).

${ }^{7}$ P. Delsing, C. D. Chen, D. B. Haviland, T. Bergsten, and T. Claeston (unpublished)

${ }^{8}$ Y. Takahide, R. Yagi, A. Kanda, Y. Ootuka, and S. I. Kobayashi, Phys. Rev. Lett. 85, 1974 (2000).

${ }^{9}$ M. Zgirski, K. P. Riikonen, V. Touboltsev, and K. Y. Arutyunov, Phys. Rev. B 77, 054508 (2008).

${ }^{10}$ F. Altomare, A. M. Chang, M. R. Melloch, Y. Hong, and C. W. Tu, Phys. Rev. Lett. 97, 017001 (2006).

${ }^{11}$ A. D. Zaikin, D. S. Golubev, A. van Otterlo, and G. T. Zimányi, Phys. Rev. Lett. 78, 1552 (1997).

${ }^{12}$ S. E. Korshunov, Sov. Phys. JETP 68, 609 (1989).

${ }^{13}$ P. A. Bobbert, R. Fazio, G. Schön, and A. D. Zaikin, Phys. Rev. B 45, 2294 (1992).

${ }^{14}$ A. Bezryadin, C. N. Lau, and M. Tinkham, Nature (London) 404, 971 (2000).

${ }^{15}$ C. N. Lau, N. Markovic, M. Bockrath, A. Bezryadin, and M. Tinkham, Phys. Rev. Lett. 87, 217003 (2001).

${ }^{16}$ A. T. Bollinger, A. Rogachev, M. Remeika, and A. Bezryadin, Phys. Rev. B 69, 180503(R) (2004).

${ }^{17}$ The upper bound marks the crossover length where finite spatialsize corrections to the thermodynamic behavior occur. To get a sense for this scale we note that $L_{\mathrm{CO}}=\hbar c / T \approx \xi \sqrt{N_{\perp}} \Delta_{0} / T$, where $\Delta_{0}$ is the BCS gap and $N_{\perp}$ is the number of transverse channels.

${ }^{18}$ The fugacity of quantum phase slips is $\zeta \sim e^{-2 c R_{Q} / R_{\xi}}$, with $c$ $\approx \frac{\pi}{16 \sqrt{3}}$ (Ref. 35). By requiring that $\zeta$ is not inhibitively small, we obtain $R_{\xi}>c R_{Q}$. For $R_{\text {tot }} \sim R_{Q}$, the length must be in the range $L \sim R_{Q} \xi / R_{\xi}<\xi / c$.
${ }^{19}$ A. T. Bollinger, R. C. Dinsmore III, A. Rogachev, and A. Bezryadin, Phys. Rev. Lett. 101, 227003 (2008).

${ }^{20}$ G. Refael, E. Demler, Y. Oreg, and D. S. Fisher, Phys. Rev. B 75, 014522 (2007).

${ }^{21}$ T. J. Rieger, D. J. Scalapino, and J. E. Mercereau, Phys. Rev. Lett. 27, 1787 (1971).

${ }^{22}$ J. Clarke, U. Eckern, A. Schmid, G. Schön, and M. Tinkham, Phys. Rev. B 20, 3933 (1979).

${ }^{23}$ J. Clarke, Phys. Rev. Lett. 28, 1363 (1972).

${ }^{24}$ D. S. Golubev and A. D. Zaikin, Phys. Rev. B 64, 014504 (2001).

${ }^{25}$ Y. Oreg and E. Demler, in Proceedings of the XXXVI Rencontres de Moriond, edited by T. Martin, G. Montambaux, and J. T. T. Van, 2001, arXiv:cond-mat/0106645.

${ }^{26}$ Y. Oreg and A. M. Finkel'stein, Phys. Rev. Lett. 83, 191 (1999).

${ }^{27}$ H. P. Büchler, V. B. Geshkenbein, and G. Blatter, Phys. Rev. Lett. 92, 067007 (2004).

${ }^{28}$ S. Sachdev, P. Werner, and M. Troyer, Phys. Rev. Lett. 92, 237003 (2004).

${ }^{29}$ G. Refael, E. Demler, Y. Oreg, and D. S. Fisher, Phys. Rev. B 68, 214515 (2003).

${ }^{30}$ P. Werner, G. Refael, and M. Troyer, J. Stat. Mech.: Theory Exp. (2005), P12003.

${ }^{31}$ J. E. Mooij and G. Schön, Phys. Rev. Lett. 55, 114 (1985).

${ }^{32}$ A. Bollinger, A. Rogachev, and A. Bezryadin, Europhys. Lett. 76, 505 (2006).

${ }^{33}$ Note that Büchler et al. (Ref. 27) considered a SC-metal transition tuned by the resistance in the measuring circuit- this results in similar physical behavior as our model.

${ }^{34}$ G. J. Dolan and L. D. Jackel, Phys. Rev. Lett. 39, 1628 (1977).

${ }^{35}$ D. Meidan, Y. Oreg, and G. Refael, Phys. Rev. Lett. 98, 187001 (2007).

${ }^{36}$ D. Meidan, Y. Oreg, G. Refael, and R. A. Smith, Physica C 468, 341 (2008).

${ }^{37}$ K. Y. Arutyunov, D. A. Presnov, S. V. Lotkhov, A. B. Pavolotski, and L. Rinderer, Phys. Rev. B 59, 6487 (1999). 